\title{
Sustainable Science? Reducing the Carbon Impact of Scientific Mega-Meetings
}

\author{
Alexandra G. Ponette-González ${ }^{{ }^{*}}$ and Jarrett E. Byrnes ${ }^{2}$ \\ Author address: ${ }^{1}$ Department of Geography, University of North Texas, 1155 Union Circle \#305279, Denton, TX 76203, USA, \\ alexandra@unt.edu
}

Author address: ${ }^{2}$ National Center for Ecological Analysis and Synthesis, Santa Barbara, CA 93101, USA

Received: September $10^{\text {th }} 2011$

Volume: 2:65-71

Published: October $29^{\text {th }} 2011$

(C) 2011 Society of Ethnobiology

\begin{abstract}
Scientists across the globe recognize the importance of reducing carbon emissions to combat climate change. At the same time, we have increased our carbon footprint through air travel to the growing number of scientific society "megameetings" that host thousands of attendees. Although alternative solutions have been proposed to reduce the environmental impact of annual conferences, these have yet to be evaluated against the business-as-usual scenario. Here, we use 9 years of annual meeting attendance data from the Ecological Society of America and the Association of American Geographers to assess the efficacy of two additional solutions: 1) alternate large national meetings that require significant air travel with smaller regional meetings that do not; and 2) incorporate geography into the meeting location selection process. The carbon footprint of annual mega-meetings ranged 3-fold, from 1196-4062 metric tons of $\mathrm{CO}_{2}$. Results indicate that an alternating schedule of national and regional meetings can reduce conference-related $\mathrm{CO}_{2}$ emissions up to $73 \%$, while improved spatial planning may result in further reductions. We discuss the benefits and tradeoffs of proposals to green scientific meetings, with a view to spark further debate on how to increase the sustainability of scientific conferences.
\end{abstract}

Key Words: Carbon Footprint, Greening the Meeting, Scientific Conferences, Planning, Sustainability

\section{Introduction}

Every year scientists showcase their research findings at large national and international conferences, some of which host thousands of participants. Regrettably, these "mega-meetings" represent a significant source of $\mathrm{CO}_{2}$ to the atmosphere. Air travel to a single meeting can generate $\sim 11,000$ metric tons of carbon dioxide (Lester 2007), while a roundtrip flight from New York City to Brussels is nearly equivalent to a Moroccan's annual $\mathrm{CO}_{2}$ emissions, 1.4 metric tons of $\mathrm{CO}_{2}$ (IEA Statistics 2010). These statistics are at odds with the values of scientists who seek to slow the current rate of $\mathrm{CO}_{2}$ increase in the atmosphere, and especially those concerned with climate change (Bonnett 2006; Young 2009; Burke 2010).

At the 2010 Dissertations Initiative for the Advancement of Climate Change Research Symposium (DISCCRS), this question arose as a topic of conversation among a small group of interdisciplinary scholars during a break-out session. In the recent scientific literature, parallel discussions and debates on how to "green meetings" reveal not only increasing concern over climate change but also a greater selfawareness among scientists at all levels of the need for a more sustainable scientific enterprise (Mills 2009; Rosenthal 2010). For example, in his editorial, Bonnett (2007) argues that to achieve sustainable conferences in the field of geography, a "cultural shift" is necessary within the discipline. Bonnett refers to the assumption of personal responsibility by academics for the environmental impacts associated with conference travel. Jarchow et al. (2011) echo this perspective for ecology and evolutionary biology, and find that raising awareness about sustainability issues at meetings is an effective means to reduce resource use among participants. However, assuming the burden of sustainability is often inconvenient Jarchow et al. 2011), and worse, may conflict with institutional norms and expectations in academia (Young 2009). As pointed out by Philippe (2008), for conferences to become sustainable, a paradigm shift must occur whereby the notion of scientific progress is decoupled from that of economic growth. 
Despite this self-reflection and awareness and a growing laundry list of proposed alternativesreduction in meeting frequency (Philippe 2008), rethinking the role of international attendance (Hall 2007), use of video- and virtual conferencing (Huang et al. 2008; Arslan et al. 2011), and purchase of carbon offsets - the benefits and tradeoffs of diverse strategies have yet to be evaluated against the business-as-usual scenario. Moreover, the efficacy of some of these measures (e.g., carbon offsets, renewable energy credits) remains highly uncertain (Struck 2010). Here, we propose two new solutions that seek to balance scientists' intellectual needs with a generous reduction in our carbon footprint: 1) alternate large national meetings that require significant air travel with smaller regional meetings that do not; and 2) incorporate geography into the meeting location selection process. According to our calculations, we find that these plans for action could more than halve conference-related $\mathrm{CO}_{2}$ emissions while maintaining the benefits provided by meetings, and even adding new ones. Additionally, our proposal reduces the carbon footprint of scientific meetings up to three times more than other suggested alternatives, including a model carbon offset program.

We present this perspective as a starting point for a deeper discussion on the sustainability of scientific conferences that is long overdue. Much like the cultural groups that are often the focal point of ethnobiological studies, interactions between scientific societies and the environment are complex and varied. Perceptions about the nature and progress of the scientific enterprise differ among societies and influence the degree to which this enterprise is, or is not, sustainably managed. In general, however, there are many questions that remain unaddressed or unresolved. Can conference attendance to megameetings grow indefinitely? What are optimal strategies for organizing sustainable conferences and how might these strategies vary by society, discipline, or specialization? What are the roles and responsibilities of individual scientists, funding agencies, and scientific societies in enhancing sustainability? And, what types of social, cultural, and institutional changes are needed to facilitate other forms of information dissemination? We hope that our proposal will contribute to a spirited and productive conversation on how to address these questions.

\section{Estimating the Carbon Footprint of Scientific Meetings}

To examine the carbon savings of multiple regional meetings versus a single national mega-meeting (hereafter referred to as "business-as-usual"), we developed two baseline emissions scenarios. We estimated $\mathrm{CO}_{2}$ emissions incurred from air and car travel to the 2002-2009 Ecological Society of America (ESA) annual meetings and to the 2010 Association of American Geographers (AAG) annual meeting by 1) members in the United States (domestic travelers), and 2) all attendees (domestic plus international travelers). We then compared baseline carbon costs to $\mathrm{CO}_{2}$ emissions resulting from US attendees driving to regional meetings. Only differences arising from changes in air and car travel were analyzed, because these comprise the bulk of conference-related $\mathrm{CO}_{2}$ emissions (Lester 2007). We considered ESA and AAG to be good candidates for analysis and representative of other large scientific societies. These mega-meetings attract considerable numbers of scientists studying climate change, and attendance is high (ESA 2009, 3599 participants; AAG 2010, 7727 attendees).

Carbon dioxide emissions under national versus regional meeting scenarios were calculated using The Conservation Fund carbon calculator (http://www.conservationfund.org). For business-as-usual estimates, address location data for all participants were compiled in a Geographic Information System (GIS) and roundtrip distances to the host city were calculated. We assumed that members located $<420$ miles $(\sim 7$ hours of driving) from the host city would drive and that international members would fly from the nearest major city (i.e., population $\geq 1$ million). It is probable that these assumptions underestimate $\mathrm{CO}_{2}$ emissions from air travel. First, we are not certain that scientists who obtain institutional funds for air travel are willing to drive to meetings even when they are located $<420$ miles from the host city. Second, we did not estimate emissions to the nearest major airport for international participants.

To estimate the carbon footprint of multiple regional meetings, we employed several driving distance models: 1) a fixed 420 mile driving distance; 2) a uniform distribution of driving distances; 3) a Poisson distribution of driving distances with a mean of 210 miles; and 4) an explicit regional geospatial model. For the geospatial model, US members were assigned to one of nine regional divisions based on the current AAG structure (www.aag.org/cs/membership/regional _divisions). Once assigned to a division, we assumed that all US members (with the exception of those in Alaska and Hawai'i) drove to a hypothetical host city randomly selected from each region. Modeled regional carbon footprints were compared to both baseline scenarios. 
We also examined the spatial distribution of US meeting attendees by zip code as well as spatial variability in the carbon cost of meetings to determine the influence of meeting location on carbon footprints. For the latter, business-as-usual $\mathrm{CO}_{2}$ emissions were divided by the total number of attendees to calculate per capita $\mathrm{CO}_{2}$ emissions for each annual conference.

Finally, we used these calculations and existing literature on the subject to approximate the $\mathrm{CO}_{2}$ reduction potential of alternate proposals. The difference between the most carbon expensive meeting location and all other meeting locations was computed to establish the range in savings that could be generated with the inclusion of per capita $\mathrm{CO}_{2}$ emissions estimates into site selection criteria. We employed the estimated contribution of international attendance to the carbon footprint of mega-meetings to evaluate the effect of decreased overseas participation on carbon dioxide emissions. The estimated annual carbon sequestration of the Society for Conservation Biology's Wild Rose Conservation Site was employed to assess the reduction potential of carbon offset projects. We reasoned that holding biennial conferences would reduce the carbon cost of scientific meetings by $\sim 50 \%$. The carbon savings potential of video- and virtualconferencing depends on the number of participants using these technologies. To estimate this, we used poll data on the willingness of scientists to participate in scientific conferences remotely.

\section{Putting a Carbon Price Tag on Business-as-Usual}

Data on number of participants, distance traveled, and $\mathrm{CO}_{2}$ emissions for ESA and AAG meetings underscore the high carbon costs associated with large annual conferences. Total attendance to the 2010 AAG annual meeting was two to three times greater than to the ESA annual meeting during any given year. Therefore, we report results for these different-sized meetings separately.

For the 2002-2009 ESA meetings, attendance ranged from 2729-4255 participants. Total distance traveled varied up to 2 -fold among meetings. Averaged over all meetings, collectively, ESA members traveled a mean $14.2 \pm 1.4$ million $\mathrm{km}$ to the conference host city. Total business-as-usual $\mathrm{CO}_{2}$ emissions ranged from 1196-2310 metric tons, with a mean carbon footprint of $1754 \pm 166$ metric tons. With the exception of the 2005 meeting held in Canada, international attendees from as many as 44 countries comprised $9-15 \%$ of the total attending population. Yet international scientists accounted for approximately one-third of the total distance traveled
(4.7 \pm 0.7 million $\mathrm{km}$ ) and contributed $25-47 \%$ of the total meeting carbon footprint.

In 2010, 7727 scientists from 65 countries attended the AAG annual meeting in Washington D.C. Combined, AAG members traveled $\sim 32$ million $\mathrm{km}$ to attend the conference, more than two times the mean distance traveled to ESA meetings. As a result of the larger size of this meeting and the greater distances involved, the 2010 AAG resulted in an estimated 4062 metric tons of $\mathrm{CO}_{2}$ emissions to the atmosphere. Compared with ESA meetings, international attendance to the AAG was much higher, accounting for $27 \%$ of the total population. International attendees comprised $56 \%$ of the total meeting carbon footprint.

On a per capita basis, $\mathrm{CO}_{2}$ emissions for the ESA meetings ranged from 0.46-0.66 metric tons. The estimated per capita AAG carbon footprint, 0.58 metric tons of carbon dioxide, fell within this range of values.

\section{Alternating National and Regional Conferences}

Depending on the model, we estimated an average 18$59 \%$ reduction in carbon emissions for multiple regional compared with national meetings (from $\sim 229$ metric tons to $\sim 730$ metric tons for ESA, from $\sim 275$ metric tons to $\sim 865$ metric tons for AAG) when only domestic travelers were considered (Figure 1a). The carbon reduction potential of an alternating schedule, however, increased to an average 49-74\% when the footprint of smaller meetings was compared to the full carbon cost of meetings with international participation (Figure 1b). Because we were unable to geocode participant address locations (i.e., identify exact geographic coordinates) for $3-10 \%$ of the sample population, our calculations underestimate the true carbon cost of large national meetings. Moreover, our regional models assumed that meeting participants do not carpool or employ public transit. Therefore, the estimated carbon savings presented here are likely quite conservative.

Our analysis also indicates that the carbon cost of national meetings varies geographically (Figure 2). For example, per capita $\mathrm{CO}_{2}$ emissions for a meeting held in Memphis, Tennessee, are 30\% lower than for a meeting held in San Jose, California. Careful selection of meeting location therefore represents a potentially simple and cost-effective way to reduce $\mathrm{CO}_{2}$ emissions. We acknowledge that holding all conferences in one or a handful of locations may not suit every scientific society. However, there are numerous ways to optimize meeting location to reduce carbon emissions: organize more meetings in areas where the majority of 


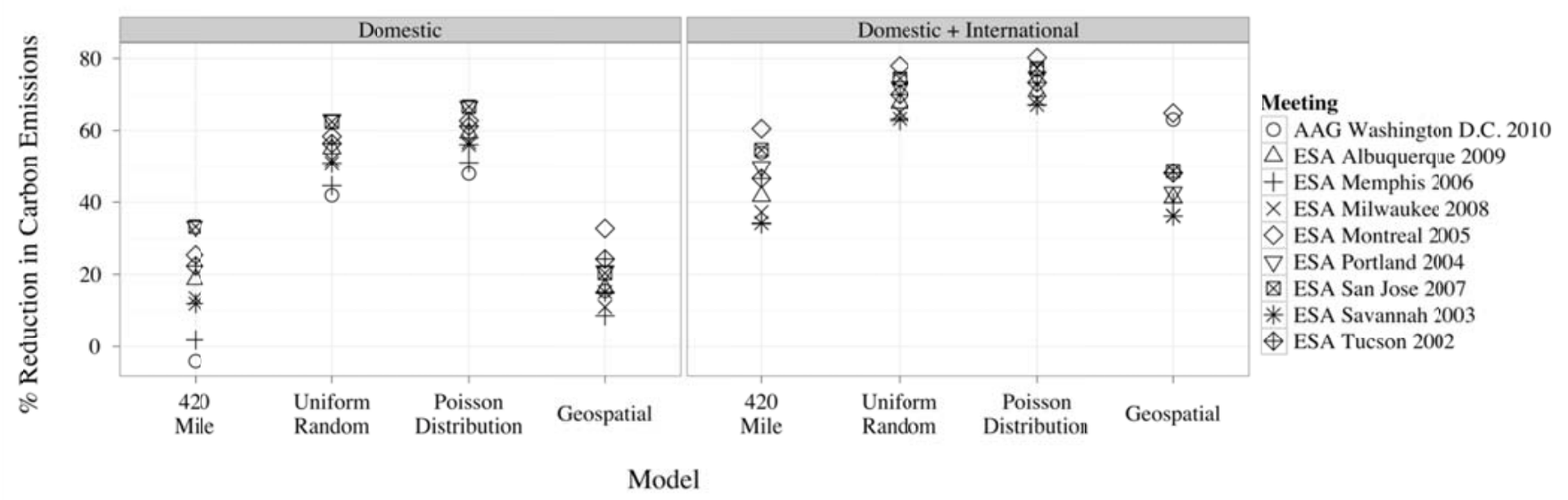

Figure 1: Percentage reduction in $\mathrm{CO}_{2}$ emissions from an alternating schedule of national and regional meetings under four different driving scenarios when the carbon footprint of regional meetings is compared to the: (a) carbon cost of domestic travel to large national meetings, and (b) carbon cost of domestic plus international travel to large national meetings. Symbols represent different national meetings used for comparisons.

the attending population resides; decrease the frequency of meetings requiring coast-to-coast travel; and hold conferences in cities that involve fewer connecting flights (Lester 2007). Overall, controlling meeting location is perhaps a secondary solution to the problem of conference-related carbon emissions. Societies will lower their emissions profile most by implementing a rotating national-regional meeting schedule.

\section{Weighing the Alternatives}

How do our solutions compare to alternative proposed solutions in terms of carbon savings? Findings show that a rotating schedule of large national and small regional meetings is up to three times more effective than other proposals (Table 1). Our analysis also reveals that inclusion of such geographic criteria as per capita $\mathrm{CO}_{2}$ emissions into the site selection process is an additional means to reduce the carbon footprint of mega-meetings.

Annual rotation of national with regional meetings could more than halve carbon dioxide emissions from conference-related travel and may provide additional benefits over those already on the table. For example, the carbon savings of our solutions are easily quantified. This stands in contrast to carbon offset projects, which are often hard to measure in terms of effectiveness (but see the Society for Conservation Biology's exemplary Carbon Offset Project). Additionally, while the purchase of carbon offsets and/or renewable energy credits may serve to increase awareness among conference attendees, these projects do not reduce emissions per se (Hall 2007) nor do they place the onus on scientists to change their travel behavior to align with the values promoted by many societies (including those covered here and the Society of Ethnobiology).

Our proposal does not require substantial financial investment and offers the advantage of maintaining the intellectual benefits of face-to-face interactions. As such, it represents a compromise between the businessas-usual approach and reduced meeting frequency. Moreover, regional meetings include enhanced opportunities for local collaboration, greater graduate and undergraduate student participation due to lower attendance cost, and increased focus on local policyrelevant issues embodying the "think globally, act locally" adage. In addition, smaller meetings are more likely to be attended by members of the public interested in engaging with scientists on regional issues of concern.

We recognize that this solution presents challenges on different levels. For individual scientists, promotion and tenure criteria typically include attendance to national and international meetings. Further, annual conferences provide an opportunity for scientists to network with distant or international collaborators. In 2010, nearly one-third of AAG participants traveled from overseas. Large meetings are ideal spaces for scientists to showcase their most important and timely research findings and to communicate with the press (McNutt 2008). And, they are often employed as recruiting grounds for students and faculty. Therefore, for some scientists, a rotating schedule may create a perverse incentive to attend other large or international meetings. For academics working in highly specialized disciplines, our approach may not be feasible. In this case, virtual or video conferencing, or "workshops 
without walls" (Arslan et al. 2011) might make more sense as a strategy to reduce carbon footprints.

Our proposal also involves tradeoffs for scientific societies or groups with broad-based memberships. An alternating schedule would involve the restructuring of scientific conferences and potentially the societies themselves. For example, while some societies have their membership concentrated in relatively few geographic areas, others are more widely dispersed. Each organization will need to examine the distribution of its own membership to decide on an optimal plan. While some societies have regional divisions (e.g., AAG) making our option immediately feasible, others do not. Implementation of regional chapters would thus require additional planning and service from society staff and members. This type of reorganization could affect society budgets and lead to decreased funds during "off years" limiting available resources for diverse non-meeting related activities. In some cases, however (e.g., the Society of Ethnobiology), conferences are not money-making enterprises, and our solution may be economically feasible and beneficial (Steve Wolverton, personal communication).

As a recent poll and commentary in Science suggest (McNutt 2008; Sills 2011), willingness to participate in conferences remotely or to attend fewer conferences is far from universal (Table 1). Under these circumstances, societies should consider incorporating per capita carbon dioxide emissions as a criterion into the meeting location selection process. There are societies including the Ecological Society of America and the Society for Conservation Biology that already calculate the footprint of annual meetings; adding this component to the site selection process could be relatively straightforward. As we have done here, geospatial technologies such as GIS can be employed in conjunction with attendance data to analyze and optimize the carbon footprint of scientific conferences. Perhaps the greatest advantage of this approach is the flexibility involved. The best optimization strategy will depend on the goals and membership of each society. Most societies have years to decades worth of meeting attendance records, data that could be utilized for a baseline analysis of the carbon cost of diverse meeting locations. In addition, the technological, software, and programming requirements are minimal, although a GIS analyst would be needed to capture, manage, and analyze the data. In the end, "adaptive management" that combines a number of approaches may be the best way to provide pragmatic, sustainable changes to conference organization.
Table 1: Benefits and drawbacks of alternative proposals to reduce the carbon footprint of scientific society meetings.

\begin{tabular}{|c|c|c|}
\hline Scenario & $\begin{array}{l}\text { Maximum } \mathrm{CO}_{2} \\
\text { reduction }\end{array}$ & Drawback \\
\hline Business-as-usual & $0 \%$ & $\uparrow \mathrm{CO}_{2}$ emissions \\
\hline Alternating schedule ${ }^{a}$ & $49-74 \%$ & $\begin{array}{l}\text { Additional } \\
\text { infrastructure and } \\
\text { planning, decreased } \\
\text { funds during "off" } \\
\text { years }\end{array}$ \\
\hline $\begin{array}{l}\text { Use of geography in } \\
\text { the selection process }\end{array}$ & $6-30 \%$ & Additional planning \\
\hline $\begin{array}{l}\text { Reduced } \\
\text { international } \\
\text { participation }^{c}\end{array}$ & $25-56 \%$ & $\begin{array}{l}\text { Reduced } \\
\text { international } \\
\text { collaboration }\end{array}$ \\
\hline Carbon offsets $^{d}$ & $23-44 \%$ & $\begin{array}{l}\text { Uncertainty } \\
\text { regarding } \\
\text { effectiveness }\end{array}$ \\
\hline $\begin{array}{l}\text { Reduction in meeting } \\
\text { frequency to biennial } \\
\text { conferences }^{\text {e }}\end{array}$ & $\sim 50 \%$ & $\begin{array}{l}\text { Fewer face-to-face } \\
\text { interactions }\end{array}$ \\
\hline $\begin{array}{l}\text { Virtual- and video- } \\
\text { conferencing }\end{array}$ & $52 \%$ & $\begin{array}{l}\text { Fewer face-to-face } \\
\text { interactions, } \\
\text { additional financial } \\
\text { investment required }\end{array}$ \\
\hline \multicolumn{3}{|c|}{ 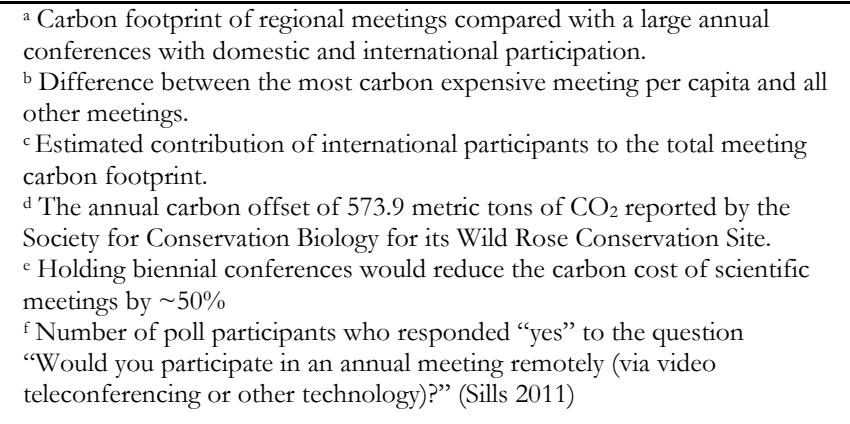 } \\
\hline
\end{tabular}

\section{Conclusion}

A formula of a rotating schedule of national and regional meetings coupled with the incorporation of a carbon-minimizing meeting selection process is feasible, and we believe that this approach could reduce carbon emissions significantly and immediately with benefits to scientific progress. As we move forward and societies and meetings grow in size and number, we believe that an ongoing dialogue on the sustainability of scientific conferences is vital. Clearly, no single solution will be applicable to all societies. Rather, a range of approaches can be used for different societies and purposes.

We also call for this debate to move beyond traditional cost-benefit analyses to a broader discussion 


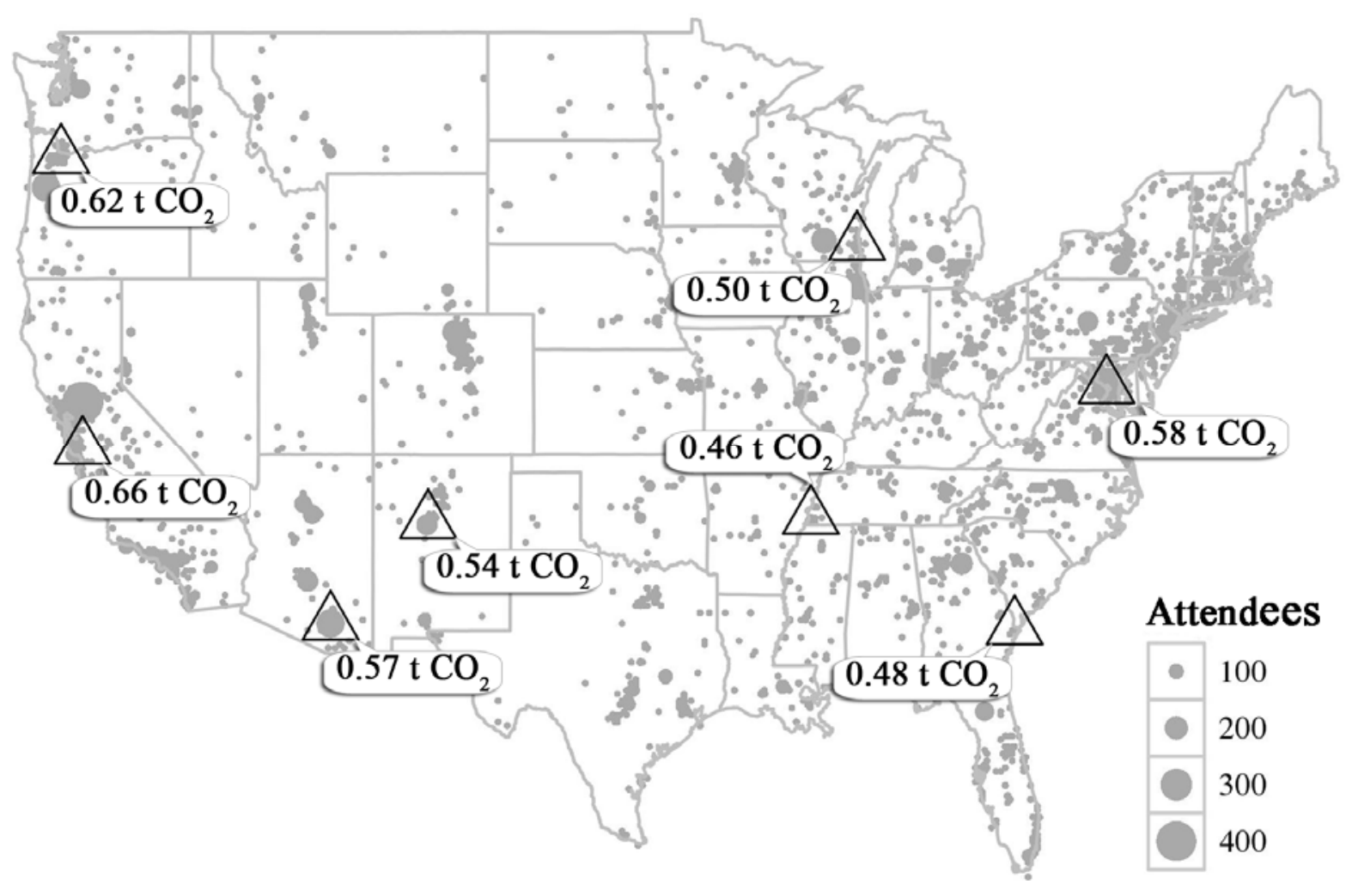

Figure 2: The carbon cost of scientific mega-meetings. Dots show the geographic distribution of attendees for all meetings evaluated within the continental United States. Dot size represents the number of attendees from any given geographic location. Triangles outline the location of national meetings considered in this analysis. Price tags indicate per capita $\mathrm{CO}_{2}$ emissions (metric tons) for national meetings.

about norms and expectations in academic culture, and how these shape our interactions, as scientists, with the environment. In their book Environmental $V$ alues in American Culture, Kempton et al. (1995:1) aptly note that "Understanding culture is an essential part of understanding environmental problems because human cultures guide their members both when they accelerate environmental destruction and when they slow it down. For everyone-leaders, citizens, and scientists alike-the cultural framework shapes the issues people see as important and affects the way they act on those issues." We specifically encourage continuing conversations on the relationship between the advancement of and growth in science; the roles and responsibilities of scientists, funding agencies, and societies in enhancing the sustainability of the scientific enterprise; environmental ethics; and the development and application of other forms of information dissemination.

\section{Acknowledgements}

This paper was developed through discussions at the DISCCRS V symposium. Symposium travel and on-site expenses were covered by the National Science Foundation through collaborative grants SES-0932916 (Whitman College, P. Yancey P.I.) and SES-0931402 (University of Oregon, R. B. Mitchell P.I.) and through a pending award from the National Aeronautics and Space Administration (Whitman College, P. Yancey P.I.). We are grateful to the Ecological Society of America and the Association of American Geographers for providing the data used in this analysis. Special thanks go to Chetan Tiwari for assistance with spatial analysis. We also thank Ronald Mitchell, Rebecca Barnes, Jennifer Marlon, Matthew Fry, and four anonymous reviewers for their comments and suggestions on this manuscript.

\section{References Cited}

Arslan, B. K., E. S. Boyd, W. W. Dolci, K. E. Dodson, M. S. Boldt, and C. B. Pilcher. 2011. Workshops without Walls: Broadening Access to Science around the World. PLoS Biology 9:1-5.

Bonnett, A. 2006. The Need for Sustainable Conferences. Area 38:229-230. 
Burke, I. C. 2010. Travel Trade-Offs for Scientists. Science 330:1476.

Hall, E. 2007. Alternative Futures for Academic Conferences: A Response to Bonnett. Area 39:125-129.

Huang, S. T., M. N. Kamel Boulos and R. P. Dellavalle. 2008. Scientific discourse 2.0. Will Your Next Poster Session be in Second Life ${ }^{\circledR}$ ? EMBO Reports 9:496499.

IEA (International Energy Agency) Statistics. 2010. $\mathrm{CO}_{2}$ Emissions from Fuel Combustion - Highlights 2010 Edition. International Energy Agency, Paris, France

Jarchow, M. E., J. W. Rice, R. M. Ritson, and S. K. Hargreaves. 2011. Awareness and convenience are important in increasing conference sustainability. Sustainability Science 6:253-254.

Kempton, W., J. S. Boster and J. A. Hartley. 1995. Environmental Values in American Culture. MIT Press, Cambridge, MA.

Lester, B. 2007. Greening the Meeting. Science 318:3638.

McNutt, M. 2008. Scientific Meetings: Worth Attending. Science 319:281.

Mills, E. 2009. Sustainable Scientists. Environmental Science \& Technology 43:979-985.

Philippe, H. 2008. Less is More: Decreasing the Number of Scientific Conferences to Promote Economic Degrowth. Trends in Genetics 24:265-267. Environment 360

Rosenthal, E. 2010. Toward Sustainable Travel: Breaking the Flying Addiction. Available at: http://e360.yale.edu/content/feature. $m s p ? i d=2280$. Accessed on September 10, 2011.

Sills, J. 2011. Travel Trade-Offs for Scientists: Readers' Poll Results. Science 331:145.

SCB (Society for Conservation Biology). The Wild Rose Conservation Site: SCB's New Carbon Offset Project for 2010-2013. Available at: http://www.conbio.org/activities/committees/Ecologi calFootprint/CarbonOffset/wildrose.cfm. Accessed on September 10, 2011.

Struck, D. Buying Carbon Offsets May Ease Eco-Guilt but not Global Warming. Available at: http://www.csmonitor.com/Environment/2010/0420 $\angle$ Buying-carbon-offsets-may-ease-eco-guilt-but-notglobal-warming. Accessed on September 10, 2011.
Young, S. 2009. Rethinking Scientific Meetings: An Imperative in an Era of Climate Change. Journal of Psychiatry and Neuroscience 34:341-34.

\section{Biosketch}

Alexandra Ponette-González is a biophysical geographer and assistant professor of geography at the University of North Texas in Denton. Her research focuses on understanding the effects of global environmental change on terrestrial ecosystems.

Jarrett Byrnes is an ecologist and postdoctoral fellow at the National Center for Ecological Analysis and Synthesis. His current research involves the effects of climate change on the network structure of food webs and the ensuing ecological consequences. 